\title{
The efficacy and safety of silodosin-a review of literature
}

\begin{abstract}
Background: Benign prostatic hyperplasia is a compilation of irritative voiding and obstructive symptoms which are consistent with reduced emptying of urine from and defective storage of urine in the bladder. Medications are a common method of treatment to delay complications and reduce symptoms. Silodosin is a highly selective alpha-1A adrenoceptor blocker that has 162 times more affinity for alpha-1a than alpha 1b, thus resulting in high uroselectivity and decreased side effects.
\end{abstract}

Aim: In this review article our aim was to elucidate the clinical effects, safety and tolerability profile of silodosin in the treatment of benign prostatic hyperplasia.

Method: Literatures were retrieved by a PubMed search, using different combinations of pertinent keywords (e.g., silodosin, hypotension, benign prostatic hyperplasia), without any limitations in terms of publication date and language. Papers which assessed the therapeutic efficacy and tolerability of silodosin were selected for inclusion according to their relevance for the topic, as judged by the authors.

Overview of clinical data: Silodosin is indicated for the treatment of the signs and symptoms of benign prostatic hyperplasia. It has a rapid onset of effect in men with lower urinary tract symptoms and improvements were seen in voiding and storage symptoms, maximum urinary flow rate and health-related quality of life. The efficacy of silodosin was maintained in several controlled studies and also non-interventional real-world setting. Silodosin was generally well tolerated.

Conclusion: Silodosin is the most uroselective $\alpha$-blocker. Silodosin has been emphasized in the 2021 European Association of Urology Conservative treatment of non-neurogenic male LUTS guidelines and it has been reported that the hypotensive effect of silodosin is comparable with placebo and has favorable safety and tolerability profile. Dosing of silodosin does not need to be adjusted according to age, concurrent medication with antihypertensives and phosphodiesterase type 5 inhibitors.

Keywords: silodosin, hypotension, benign prostatic hyperplasia, lower urinary tract symptoms

\author{
Volume 9 Issue 6 - 202I
}

\author{
Kubra Saygisever-Faikoglu,' Gokhan \\ Faikoglu, ${ }^{2}$ Fatmanur Otmar Ozcan, ${ }^{3}$ Barkin \\ Berk $^{4}$ \\ 'Department of Pharmacology, Cerrahpasa Faculty of Medicine, \\ Istanbul University, Turkey \\ ${ }^{2}$ Department of Medical Pharmacology, Faculty of Medicine, \\ Beykent University, Turkey \\ ${ }^{3}$ Department of Internal Medicine, Okmeydani Training and \\ Research Hospital, Turkey \\ ${ }^{4}$ Department of Pharmaceutical Chemistry, School of Pharmacy, \\ Istanbul Medipol University, Turkey
}

Correspondence: Gokhan Faikoglu, Department of Medical Pharmacology, Faculty of Medicine, Beykent University, Istanbul, Turkey, Email gokhan.faikoglu@gmail.com

Received: November 29, 2021 | Published: December 16,
2021
Abbreviations: BOOI, bladder outlet obstruction index; BPE, benign prostatic enlargement; $\mathrm{BPH}$, benign prostatic hyperplasia; $\mathrm{CI}$, confidence interval; $\mathrm{CT}$, computerized tomography; $\mathrm{ED}$, erectile dysfunction; F-URS, flexible ureterorenoscopy; CGIC, clinical global impression change; IELT, intravaginal ejaculation latency times; IPSS, international prostate symptom score; LUTS, lower urinary tract symptoms; LUTS/BPO, lower urinary tract symptoms/ benign prostatic obstruction; MCC, maximum cystometric capacity; PVR, postvoid residual urine volume; Rej, retrograde ejaculation; TUIP, transurethral incision of the prostate; Qmax, peak urinary flow rate; QoL, quality of life; OABSS, overactive bladder symptom score

\section{Introduction}

Benign prostatic hyperplasia (BPH) is closely related to aging. Although it is not life-threatening, its clinical manifestation as lower urinary tract symptoms (LUTS) can affect the patient's quality of life. ${ }^{1} \mathrm{Up}$ to $30 \%$ of men older than 65 may experience distressing LUTS. BPH is a compilation of irritative voiding and obstructive symptoms which are consistent with reduced emptying of urine from and defective storage of urine in the bladder. ${ }^{2}$ Medications are a common method of treatment to delay complications and reduce symptoms. Renal failure, bladder diverticula, bladder stones, urinary retention, urosepsis, infections, hematuria, urinary incontinence are complications associated with untreated $\mathrm{BPH} .{ }^{3,4}$
During the histologic examination of the disease, only about $50 \%$ of patients develop an enlarged prostate and $25 \%$ of patients show symptoms of clinical voiding dysfunction. Voiding symptoms are consistent with BPH likely in $8 \%$ of men 40 years old and rising up to $35 \%$ of men between 60 to 69 years old. It was reported that about $30 \%$ of all male patients who live to the age of 80 years will need a prostatectomy for serious voiding symptoms of BPH. ${ }^{1}$

In a survey of 19.165 people from general population $64.3 \%$ of participants reported at least one LUTS. The prevalence has increased to $80.7 \%$ in men $\geq 60$ years old. ${ }^{5}$ According to the pooled prevalence data from USA, UK and Sweden $76.3 \%$ of women $72.3 \%$ of men and had at least one LUTS in their life. ${ }^{6}$ Orthostatic hypotension, dizziness, tiredness are the common advers effects with alpha-blocker therapy. Uroselective alpha-blocker therapy that may cause fewer advers effects seem to be safer than non-selective ones. ${ }^{4}$

Alpha-1 receptor blocker drugs are the first and most common molecules in urology practice in the treatment of LUTS associated $\mathrm{BPH}$. Prostate stroma and bladder neck are rich in alpha receptors. In normal conditions the stroma and epithelial tissue ration is $2 / 1$, but in the early stages of BPH this ratio changes to $4 / 1$. The relaxation of stroma via alpha receptor blockers in BPH patients will decrease the resistance on prostatic urethra thus leveraging the improvement of complaints. $^{7,11}$ 
First short-acting drug prazosin has been used in the treatment of LUTS. However due to its short half life and frequent dosing schedule it was not a common preference. Later, both selective and long-acting agents have been developed. Alpha-1 receptor antagonists have been divided into 2 classes as uroselective and non-selective. There are also subtypes such as alpha-1a receptors especially found in prostate, alpha-1b receptors found in vascular system, central nervous system, spleen and lung and alpha-1 d receptors found in the bladder and spinal cord. ${ }^{8}$

Alpha-1 adrenergic receptor antagonists are the major treatment of LUTS and BPH. Silodosin is an $\alpha-1 \mathrm{~A}$ (Alpha-1A) adrenergic receptor antagonist that is effective, safe and clinically useful in the treatment of LUTS associated with BPH. ${ }^{9}$ Silodosin is a highly selective alpha-1a adrenoceptor blocker that has 162 times more affinity for alpha-1a than $1 \mathrm{~b}$, thus resulting in high uroselectivity and decreased side effects. Mostly these receptors are in the prostate and urethra. Due to blood pressure-related adverse effects caused by $\alpha-1 \mathrm{~B}$, significant improvements were seen in maximum urinary flow rate and International Prostate Symptom Score with silodosin. ${ }^{10}$

Alpha-1 $(\alpha 1)$ adrenergic antagonists (silodosin) are preferred over $5 \alpha$-reductase inhibitors due to their fast onset of action and $5 \alpha$-reductase inhibitors are often utilized with an $\alpha 1$-adrenergic antagonist due to the delay in clinical effect. It also has regulatory potential in blood pressure and it has proven to be effective as medical expulsive therapy. ${ }^{9}$

According to the results of meta-analysis studies, alpha-1 receptor antagonists create an increase of $20-40 \%(2-3 \mathrm{~mL} / \mathrm{sec})$ in urinary flow rate. This increase is 10-15 times greater than the improvement with placebo. The symptomatic improvement was 30-50\% with alpha-1 receptor blockers (4-6 points) whereas with placebo this improvement was only $10-20 \% .{ }^{11,12}$ These drugs are not curative, they provide symptomatic improvement. The relationship between efficacy of alpha-1 receptor blockers with prostate volume is another controversial issue. In some studies the efficacy of of alpha-1 receptor blockers efficacy was found independent from prostate volume. According to European Association of Urology (EAU) guideline, the volume of prostate is non-significant in treatment regimens less than a year. In terms of long term medication if prostate volume is below 40 $\mathrm{mL}$, pharmacotherapy has been more effective. ${ }^{12}$

\section{Overview of silodosin data}

\section{Pharmacologic properties}

The $\alpha-1 \mathrm{~A}$ adrenoceptor antagonists are member of the $\mathrm{G}$ protein-coupled receptors (GPRC) family. Binding of adrenaline and noradrenaline leads to the activation of phospolipase $\mathrm{C}$ and the formation of second messengers like inositol triphosphate and diacylglycerol. Ultimately calcium level increases involved in muscle-cell contraction. ${ }^{13}$

Silodosin is the member of the ABs family and has highest $\alpha 1 \mathrm{~A}$ -AR/ $1 \mathrm{~B}$-AR affinity ratio. This original pharmacological properties of silodosin lead to urodynamic and clinical effects. Advantages of pharmacological uroselectivity profile that characterize silodosin among other ABs include highest level of BOOI improvement and placebo-like cardiovascular AE. ${ }^{7}$ There is a positive correlation between $\mathrm{BOOI}$ improvement and $\alpha 1 \mathrm{~A} / \alpha 1 \mathrm{~B}$ receptor affinity ratio (Table 1).
Table I Mean BOOI improvements of $A B s$ in patients with LUTS/BPO ${ }^{7}$

\begin{tabular}{lc}
\hline Mean BOOI improvements of ABs in patients with LUTS/BPO \\
\hline Silodosin & -30.45 \\
Doxazosin & -19.41 \\
Alfuzosin & -14.88 \\
Tamsulosin & -14.27 \\
Terazosin & -6.69 \\
\hline
\end{tabular}

Terazosin, doxazosin and alfuzosin are non-selective agents. Tamsulosin is selective for the $\alpha 1 \mathrm{~A}$ and $\alpha 1 \mathrm{D}$ subtypes. In addition silodosin is highly selective and very strong affinity for the $\alpha 1 \mathrm{~A}$ adrenergic receptors (162 times higher than that for the $\alpha 1 \mathrm{~B}$ adrenergic receptors and 55 times higher than that for the $\alpha 1 \mathrm{D}-\mathrm{AR}){ }^{7}$ Silodosin is absorbed rapidly and $97 \%$ bound to plasma proteins. Silodosin is extensively metabolized via CYP3A4, glucuronidation, and alcohol and aldehyde dehydrogenase pathways. Elimination half-life (t1/2) of silodosin is approximately 13 hours and $(\mathrm{t} 1 / 2)$ of its metabolites approximately 24 hours in healthy volunteers thus allowing a convenient dosing schedule. ${ }^{13,14}$

As the prevalance of BPH increases with age once daily dosing of silodosin $8 \mathrm{mg}$ can be safely used in elderly patients without any titration. ${ }^{15}$ According to FDA (U.S. Food and Drug Administration) recommendations silodosin is contraindicated in patients with severe hepatic and renal impairment. ${ }^{16}$ Once-daily dosing with $8 \mathrm{mg}$ silodosin does not need to be adjusted according to age, uroselectivity, concurrent medication with antihypertensive and phosphodiesterase type 5 inhibitors. ${ }^{17}$

\section{Clinical data}

A randomized double-blind study with more than 800 patients is conducted by Montorsi et al. (2010). Total IPSS, storage values and voiding lower values were found to be at least as effective as tamsulosin for silodosin. It is much more effective than tamsulosin in causing concurrent improvement of bothersome LUTSs such as frequency, unfinished emptying and nocturia $(p<0.05)$. Treatment with silodosin $8 \mathrm{mg}$ QD has shown that it is safe according to data collected in 1581 patients. The most common reported adverse reaction was retrograde ejaculation $(21.5 \%$ of patients in placebocontrolled trials vs $0.8 \%$ with placebo) which has led to therapy termination in only $3.9 \%$ of patients. ${ }^{16}$

Silodosin is the most uroselective $\alpha$-blocker known (34), is more effective than tamsulosin in the treatment of nocturia ${ }^{16}$ and relieving the most bothersome symptoms of BPH (frequent urination, nocturia, feeling of incomplete emptying of the bladder). ${ }^{16}$ Silodosin has a fast onset of action (2-6 hours) meaning that the efficacy starts within the first dose and first day of treatment. ${ }^{18}$

In a publication dealing with Cochrane database covering 4.295 patients involving the age group and prostate volume that was usually observed, the evaluation of International Prostate Symptom Score (IPSS), revealed that silodosin was significantly more effective than placebo. ${ }^{59}$ Cardiovascular and treatment discontinuing side effects were almost the same as placebo. In a systematic review of 23 randomized controlled trials conducted in 2018, the data of 9.000 patients were reviewed and silodosin has been shown to provide significant improvement in both IPSS and QoL (quality of life). ${ }^{60}$ 
The drug switch rate is significantly lower with silodosin than other $\alpha$-blockers. ${ }^{25}$ Silodosin can be used safely with PDE-5 inhibitors and antihypertensive drugs without the risk of orthostatic hypotension. ${ }^{19,25}$ Regarding this the favorable cardiovascular safety and long term usage should be emphasized..$^{25}$

According to the European Association of Urology (EAU) 2021 Guidelines; there is a statistically significant increase in the risk of developing a vascular event compared to placebo with the use of alfuzosin, terazosin and doxazosin. ${ }^{12}$ The incidence of hypotension associated with silodosin therapy was similar to placebo. ${ }^{12}$ Silodosin can be used in combination with phosphodiesterase-5 inhibitors in patients with erectile dysfunction without causing clinical orthostatic changes and safely without the need for any dose adjustment (Table 2). ${ }^{19}$

Table 2 Efficacy of silodosin in compared to placebo in 3 different trials ${ }^{16}$

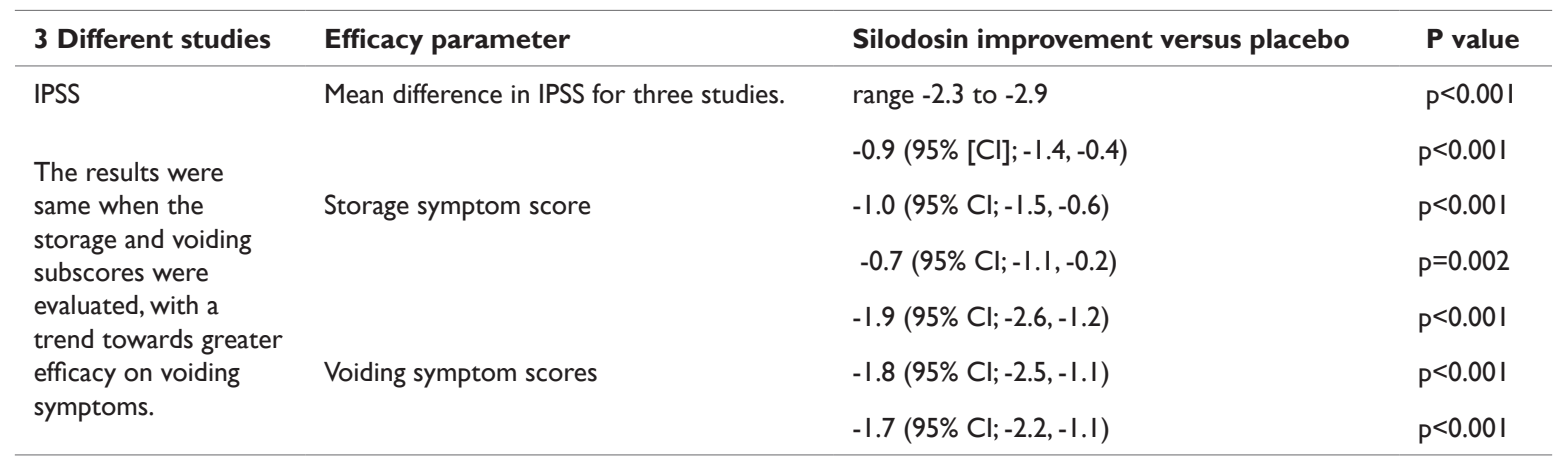

In two phase III studies silodosin compared to placebo treatment induced rapid (within 3 to 4 days) recovery in IPSS (-1.9); irritative $(-0.5)$ and obstructive (-1.4) subscores significantly. The IPSS change versus baseline was $-4.2 \pm 5.3$ for silodosin and significantly better than placebo. Qmax improvement was observed in 2 to 6 hours after the first administration. Only $2.8 \%$ of patients taking silodosin have terminated the treatment due to adverse events. The rate of orthostatic hypotension were comparable for silodosin and placebo $2.6 \%$ and $1.5 \%$, respectively. ${ }^{18}$ The most common adverse reactions reported were the same reactions that caused the discontinuations: retrograde ejaculation $(21.5 \%$ of patients in placebo-controlled trials vs $0.8 \%$ with placebo; $23.6 \%$ overall).

According to a previous article by MacDiarmid et al. (2010) silodosin was co-administrated with maximum doses tadalafil or sildenafil and no important pharmacodynamic interaction has been observed. ${ }^{19}$ Safety risks of increased heart rate or orthostatic hypotension were not associated with concurrent administration. Silodosin may be a proper treatment of $\mathrm{BPH}$ and erectile dysfunction with sildenafil or tadalafil. ${ }^{20}$

Capitanio et al. (2013) published a review and stated that orthostatic hypotension did not increase with concomitant use of silodosin with antihypertensives. Silodosin can be taken with phosphodiesterase type 5 inhibitors concomitantly. Patients showed better improvement when switching tamsulosin to silodosin than silodosin to tamsulosin. ${ }^{21}$ The clinical symptoms of patients that suffer from nocturia, ureteral stone passage, prostatitis, pelvic pain syndrome have also ameliorated with the use of silodosin. According to Capitanio et al. (2013) silodosin was efficacious for the initial treatment of LUTS with a cardiovascular safety profile. ${ }^{21}$

Manohar et al. (2017) conducted a double-blind, randomized trial comparing silodosin compared with tamsulosin or alfuzosin. Silodosin improved IPSS (11.7 \pm 4.18$)$, QoL (2.2 \pm 0.76$)$, Qmax (2.2 \pm 0.76$)$ significantly better than tamsulosin or alfuzosin in the first week of the trial..$^{22}$ The superiority of silodosin to other alpha-1 blockers was demonstrated with various parameters. While the baseline IPSS mean score was $20.0 \pm 4.4$, this score decreased significantly at week 8 to
$18.6 \pm 4.5(\mathrm{p}<0.03)$. A significant improvement in QoL was observed after switching to silodosin from baseline $(\mathrm{p}<0.001) .{ }^{23}$ Tamsulosin was used $0.4 \mathrm{mg}$ once daily for at least 12 months then the non-responders have been switched to $8 \mathrm{mg}$ silodosin once a day (Table 3$){ }^{23}$

Table 3 Efficacy of silodosin in patients who were switched from tamsulosin ${ }^{23}$

\begin{tabular}{llll}
\hline & Mean change & $\mathbf{9 5 \%} \mathbf{C l}$ & $\mathbf{P}$ \\
\hline IPSS & $(-1.3) \pm(1.4)$ & $(-1.6)-(-1.0)$ & $<0.03$ \\
QoL & $(-0.8) \pm(1.0)$ & $(-1.0)-(-0.6)$ & $<0.001$
\end{tabular}

The discontinuation rate of silodosin has been found $1.3 \%$. Silodosin when compared to tamsulosin did not cause any significant change in heart rate and systolic-diastolic blood pressure from baseline; but tamsulosin produced a significant drop in systolic blood pressure (BP). ${ }^{24}$ In conclusion, there were improvements in QoL (quality of life) and IPSS score in patients with BPE who did not respond to tamsulosin treatment for at least 12 months. ${ }^{23}$

Kim et al. (2014) conducted an observational study on the prescription changes of 3200 patients due to adverse events and lack of efficacy of $\alpha 1$-blockers. The three main reasons for shifting the $\alpha 1$ blocker prescriptions were efficacy problems (52.7\%), adverse events (33.1\%) and high costs (7.0\%). Among $\alpha 1$-blockers silodosin has the lowest (statically significant) rate of prescription change compared to tamsulosin, doxazosin and alfuzosin. The percentage of prescription change $(16.3 \% ; \mathrm{p}<0.05)$ and hemodynamic adverse events $(2.4 \%$; $\mathrm{p}<0.006)$ were significantly lower for silodosin compared with other three members. ${ }^{25}$

In a multicenter randomized trial Yamaguchi K. et al. (2013) showed that alpha-1A selective blockers (silodosin) are more effective in improving voiding symptoms than alpha-1D (naftopidil). ${ }^{26}$ In a randomized prospective study involving 120 outpatients with untreated BPE associated with urinary urgency at least once per week and OABSS of $\geq 3$. Long-term combination treatment with silodosin and propiverine (With 1 year treatment of silodosin $8 \mathrm{mg} /$ day and propiverin $20 \mathrm{mg} /$ day) was effective and safe for BPE patients with voiding and $\mathrm{OAB}$ symptoms (Table 4$).{ }^{27}$ 
Table 4 The changes in subjective symptoms between the two groups ${ }^{27}$

\begin{tabular}{|c|c|c|c|}
\hline & silodosin & silodosin+propiverine & $\mathbf{P}$ (inter-group) \\
\hline IPSS-QOL (I year) & 3.6_I.2 (_I.2) & 3.0_I.2(_I.9) & $P=0.01$ \\
\hline OABSS (I year) & $5.2 \_2.6(2.4)$ & $4.2 \_2.2(3.4)$ & $P=0.04$ \\
\hline OABSS-urgency (I year) & I.8_I.2(_I.2) & I.2_I.0(_I.8) & $P=0.006$ \\
\hline MCC (ml) (I year) & $248 \pm 50(s ̦ 33)$ & $269 \pm 92(+6 I)$ & $P=0.02$ \\
\hline PVR (ml) (I year) & $35 \pm 35(-17)$ & $66 \pm 54(+20)$ & $\mathrm{P}=<0.001$ \\
\hline
\end{tabular}

\section{Additional treatment areas of silodosin}

Administration of silodosin before flexible ureterorenoscopy (F-URS) procedure reduces entrance to the bladder, entrance to ureteric orifice and access sheath time. ${ }^{28}$ Taking $8 \mathrm{mg}$ silodosin 3 hour before intercourse it is a potential on demand oral contraceptive for males and it is also reversible. In a one year, double-blind randomized placebo-controlled study of Bhat et al. (2020) it was observed that unintended pregnancies in the female partners with proven fertility were prevented. Post ejaculation urine and masturbation semen were analyzed for spermatozoa and there was no drop out due to adverse events. $^{29}$

In a phase IV multicenter clinical trial that evaluated the efficacy and safety of silodosin the results suggested that silodosin is safe and effective to reduce the episodes of nocturia in benign prostatic hyperplasia patients. Ejaculatory disorder (7.6\%) was the only significant common adverse reaction. The IPSS, overactive bladder symptom score and nocturia scores were improved by using silodosin for 12 weeks as $8 \mathrm{mg}$ once daily. ${ }^{30}$ LUTS is not specific for BPH, neurogenic bladder and other urologic disorders including prostate cancer or prostatitis can also cause LUTS. Moon et al. (2015) investigated the efficacy of silodosin in voiding dysfunction cases with neurogenic bladder. They found that silodosin has positive effects on IPSS-QoL score, Qmax and IPSS in patients with neurogenic bladder. Silodosin was evaluated as a good, safe and effective treatment for neurogenic voiding dysfunction. ${ }^{31}$

In the same study a total of 43 women and 39 men (mean age $59.7 \pm 12.9$ years) were evaluated. Brain lesions have been documented in $42.7 \%$ and spinal cord diseases have been documented in $30.5 \%$ and peripheral nervous system diseases have been documented in $17.1 \%$ cases. $^{31}$ Total mean IPSS decreased significantly $(\mathrm{P}=0.0002)$ after twelve weeks of silodosin treatment from $22.23 \pm 6.80$ (baseline) to $14.98 \pm 9.48$. Significant decrease was also observed in IPSS-QoL score from $4.62 \pm 0.92$ to $3.48 \pm 1.63(\mathrm{P}<0.0001)$. Qmax increased significantly from $10.72 \pm 2.66$ to $15.14 \pm 6.63 \quad(\mathrm{P}<0.0001)$, while PVR decreased but did not reach statistical significance. Total IPSS, voiding and storage symptom score, IPSS-QoL score and Qmax were noticeably improved in both women and men (Table 5). ${ }^{31}$

Table 5 Efficacy of silodosin on IPSS total scores, IPSS QoL scores, Qmax and PVR ${ }^{31}$

\begin{tabular}{|c|c|c|c|c|c|c|c|c|c|}
\hline & $P$ value & $\begin{array}{l}\text { Men Vs. } \\
\text { Women }\end{array}$ & $\begin{array}{l}\text { After } 12 \\
\text { weeks }\end{array}$ & Baseline & & $\begin{array}{l}\text { After } 12 \\
\text { weeks }\end{array}$ & Baseline & $P$ value & $\begin{array}{l}\text { Men Vs. } \\
\text { Women }\end{array}$ \\
\hline \multirow[t]{2}{*}{ IPSS total } & $<0.001$ & $>0.05$ & $14.98 \pm 9.48$ & $22.23 \pm 6.80$ & Voiding & $8.98 \pm 6.08$ & $14.16 \pm 4.34$ & $<0.001$ & $>0.05$ \\
\hline & & & & & Storage & $6.00 \pm 4.41$ & $8.07 \pm 4.05$ & $<0.001$ & $>0.05$ \\
\hline IPSS-QoL & $<0.001$ & $>0.05$ & $3.48 \pm 1.63$ & $4.62 \pm 0.92$ & & & & & \\
\hline $\mathrm{Qmax}(\mathrm{mL} / \mathrm{sec})$ & $<0.001$ & $>0.05$ & $15.14 \pm 6.63$ & $10.72 \pm 2.66$ & & & & & \\
\hline PVR (mL) & 0.736 & $>0.05$ & $45.09 \pm 70.69$ & $47.51 \pm 53.16$ & & & & & \\
\hline
\end{tabular}

\section{Effect of silodosin on premature ejaculation}

According to Sato et al. (2017) silodosin (4 mg) was even more effective than naftopidil $(25 \mathrm{mg})$ in the treatment of premature ejaculation. ${ }^{33}$ They have conducted a study of 26 individuals with a mean age of 50.7 (21-76) years who reported premature ejaculation for an average of 6 years (1-18). ${ }^{33}$ Patients used silodosin $4 \mathrm{mg}$ or naftopidil $25 \mathrm{mg} 1$ hour before sexual intercourse, alternating drugs at least three times each. The change in clinical overall impression for premature ejaculation, premature ejaculation profile, and intravaginal ejaculation latency was evaluated at baseline and during treatment. Silodosin significantly prolonged intravaginal ejaculation latency compared to baseline and naftopidil $(\mathrm{p}<0.01)$. Mean intravaginal ejaculation latency times at baseline, control and with silodosin were $1.9,4.1$, and 7.6 minutes respectively (Table 6)..$^{33}$

Table 6 Efficacy of silodosin versus naftopidil in the treatment premature ejaculation ${ }^{33}$

\begin{tabular}{|c|c|c|c|c|}
\hline & Silodosin & Naftopidil & $\begin{array}{l}\text { Silodosin versus } \\
\text { naftopidil }\end{array}$ & $\begin{array}{l}\text { Silodosin versus } \\
\text { baseline }\end{array}$ \\
\hline CGIC improvement & $92.3 \%(\mathrm{Cl} 0.78-0.97)$ & $46.1 \%(\mathrm{Cl} 0.24-0.64)$ & $P=0.0002$ & $\begin{array}{l}\text { more improvement than } \\
\text { naftopidil }\end{array}$ \\
\hline \multirow[t]{2}{*}{ Mean IELT } & 7.6 minute & 4.I minute & $P<0.01$ & $P<0.01$ \\
\hline & & & 7.6 vs 4.1 minute & 7.6 vs 1.9 minute \\
\hline
\end{tabular}


Table Continued...

\begin{tabular}{|c|c|c|c|c|}
\hline & Silodosin & Naftopidil & $\begin{array}{l}\text { Silodosin versus } \\
\text { naftopidil }\end{array}$ & $\begin{array}{l}\text { Silodosin versus } \\
\text { baseline }\end{array}$ \\
\hline $\begin{array}{l}\text { Distress as a result of decreased } \\
\text { semen volume }\end{array}$ & $54 \%$ & $46 \%$ & $P=0.3635$ & $<0.0007$ \\
\hline Improvement in ejaculation control & $\begin{array}{l}\text { best outcomes vs naftopidil } \\
\text { and baseline }\end{array}$ & improve vs baseline & $P=0.025$ & $P<0.0001$ \\
\hline $\begin{array}{l}\text { Satisfaction with sexual intercourse } \\
\text { improvement }\end{array}$ & $\begin{array}{l}\text { best outcomes vs naftopidil } \\
\text { and baseline }\end{array}$ & improve baseline & $P=0.0134$ & $<0.0001$ \\
\hline Difficulty in relationship with partner & no significant change & no significant change & $P=0.703$ & 0.2536 \\
\hline
\end{tabular}

Roehrborn et al., ${ }^{34}$ performed a double blind controlled study and utilized $8 \mathrm{mg}$ silodosin $(\mathrm{n}=466)$ once daily and the placebo group $(\mathrm{n}=457)$ placebo once daily for 12 weeks. IPSS and Qmax improvement were greater in the silodosin $\mathrm{REj}+$ and $\mathrm{REj}$ - vs placebo. $\mathrm{REj}+$ group had greater improvement in symptoms and the Qmax vs $\mathrm{REj}$ - group. High rate of $\mathrm{REj}+$ is attributed to LUTS treatment with efficacy of silodosin (Table 7). ${ }^{34}$ Sertkaya et al. (2019) elaborated the sexual nocebo effects of silodosin. They reported that there are some nocebo effects like reported low semen volume $(0,04)$. In the same study $7 \%$ of patients discontinued the treatment due to anejaculation side effects. ${ }^{35}$

Table 7 Treatment efficacy in patients with and without silodosin-related retrograde ejaculation ${ }^{34}$

\begin{tabular}{|c|c|c|c|c|c|}
\hline & $\begin{array}{l}\text { Placebo (change from } \\
\text { baseline) in } 12 \text { weeks (mean) }\end{array}$ & Silodosin & $\begin{array}{l}\text { Silodosin (change from } \\
\text { baseline) in } 12 \text { weeks (mean) }\end{array}$ & $\begin{array}{l}\text { P value (silodosin } \\
\text { vs placebo) }\end{array}$ & $\begin{array}{l}\text { P value } \\
\text { (REj+ vs REj-) }\end{array}$ \\
\hline \multirow{2}{*}{ IPSS } & \multirow{2}{*}{$-3.5(5.8)$} & REj+ & $-7.2(7.2)$ & $<0.0001$ & \multirow{2}{*}{$=0.3856$} \\
\hline & & REj- & $-6.1(6.4)$ & $<0.0001$ & \\
\hline \multirow{2}{*}{$\begin{array}{l}\text { Qmax, ml/ } \\
\text { sec }\end{array}$} & \multirow{2}{*}{ I.5 (4.4) } & REj+ & $3.1(4.8)$ & 0.0003 & \multirow{2}{*}{0.0699} \\
\hline & & REj+ & $2.4(4.3)$ & 0.0184 & \\
\hline
\end{tabular}

\section{Silodosin and stone expulsion via surgical intervention}

The efficacy of silodosin $8 \mathrm{mg}$ once daily in patients over 50 years of age (caucasian) has been evaluated in bladder outlet obstruction. Significant reduction (from 70.6 to 39.2 ) in mean bladder outlet obstruction index has been detected in $53.3 \%$ of the cases. Surgery was delayed due to the urodynamic improvements. ${ }^{36}$ Distal ureteral calculi (max $10 \mathrm{~mm}$ stone size) expulsion time was very short with silodosin and has been discontinued after the expulsion. It is a short, safe, effective and more rational treatment choice than invasive or other medical methods. ${ }^{37}$ In different studies similar results have been denoted that stone expulsion rate was significantly better in silodosin $(80.3 \%)$ than tamsulosin $(61.2 \%)$; first week rates were also better in silodosin $(47.1 \%$ vs $31.7 \%) .{ }^{38}$ In another study by Kumar et al. (2015) calculi expulsion rate for silodosin was better $(83.3 \%)$ than tamsulosin $(64.4 \%)$ and tadalafil $(66.7 \%)$; and also lower time of expulsion (silodosin versus tamsulosin $\mathrm{p}=0.006$ ) and (silodosin versus tadalafil $\mathrm{p}=0.016$ ). The authors declared that silodosin has better control of pain and lesser adverse events and colicky episodes. ${ }^{39}$

In a randomized double blinded controlled trial by Wang et al. (2016) stone expulsion period was $6.3 \pm 2.1$ days $(\mathrm{P}<0.001)$ and expulsion rate was $77.4 \%$. $(\mathrm{p}=0.006)$. Analgesic consumption was significantly lower $(255.97 \pm 112.48 \mathrm{p}<.0001)$ in silodosin group..$^{40}$ In some cases distal ureteric stones were detected by ultrasonography and $\mathrm{CT}$ before the initiation of medical expulsive therapy. The results showed that silodosin $(82.4)$ was significantly $(p=0.007)$ better than tamsulosin $(61.5 \%)$ in expulsion rate and time $(9.4 \pm 3.8$ days vs $12.7 \pm 5.1$ days; $p=0.001) .{ }^{42}$ Regarding all these studies one can derive that management of distal ureteric stones with shock wave lithotripsy and ureteroscopy is expensive and comprimises certain risks such as postoperative stricture, perforation and ureteric avulsion. ${ }^{42}$
In a prospective randomized controlled trial by Bayar et al. (2020) silodosin has been compared with mirabegron in aspect of expulsive therapy for ureteral stones. This study suggested that: the interval of stone expulsion in patients with distal localisation and the interval of stone expulsion in patients with stone size smaller than $6 \mathrm{~mm}$ were shorter in the silodosin group than the control group. The stone expulsion interval was not affected by mirabegron thus indicating silodosin was better for the therapy of ureteric stone expulsion in adults (Table 8$){ }^{43}$

Table 8 Silodosin has been compared with mirabegron in aspect of expulsive therapy for ureteral stones ${ }^{43}$

\begin{tabular}{llll}
\hline \multicolumn{4}{l}{ Stone expulsion interval } \\
\hline & Silodosin & Control group & P value \\
\hline Expulsion & $7.1 \pm 4.5$ days & $12 \pm 8.7$ & $\mathrm{P}=0.034$ \\
$\begin{array}{l}\text { Stone size }<6 \mathrm{~mm} \\
\begin{array}{l}\text { Analgesic } \\
\text { requirement }\end{array}\end{array}$ & $5.8 \pm 4$ & $12.2 \pm 2.8$ & $\mathrm{P}=0.004$ \\
\hline
\end{tabular}

\section{Medical expulsive therapy in children}

Silodosin that is a well-known $\alpha$-blocker for medical expulsive therapy has also been investigated to evaluate the distal ureteric stones in children.

In a prospective, single-blind, placebo-controlled, randomized study by Soliman et al. (2021), 167 children with distal ureteric stone less than $1 \mathrm{~cm}$ have been enrolled in the study. ${ }^{61}$ Patients were divided into 3 groups. Patients of group I received silodosin $(4 \mathrm{mg})$, group II received tamsulosin $(0.4 \mathrm{mg})$ and group III had placebo 
once daily. The stone expulsion rate was significantly higher and the time to stone expulsion was significantly shorter in group I $(89.3 \%$, $12.4 \pm 2.3$ days $)$ and group II $(74.5 \%, 16.2 \pm 4.2$ days $)$ compared to group III $(51.8 \%, 21.2 \pm 5.6)$. Silodosin performed significantly better stone expulsion rate and shorter expulsion time than tamsulosin for treatment of distal ureteric stone. Both medications showed good safety profiles in children. ${ }^{61}$ Elgalaly et al. (2017), conducted a study on 40 paediatric patients ( 27 boys and 13 girls) diagnosed with unilateral, single, radiopaque distal ureteric stone of $<10 \mathrm{~mm}$ (mean age 8.1 years). ${ }^{62}$ The patients were randomly divided into two groups: Group A, received silodosin $4 \mathrm{mg}$ as a single bedtime dose; and Group B, received placebo as a single bedtime dose. Ibuprofen was prescribed to both groups on-demand for pain episode relief. The mean stone expulsion time was 7.0 versus 10.4 days in groups $\mathrm{A}$ and $\mathrm{B}$, respectively $(\mathrm{P}=0.02)$. The mean number of pain episodes requiring ibuprofen was 2.3 versus 4.7 episodes in groups $\mathrm{A}$ and $\mathrm{B},(\mathrm{P}<0.001)$. According to the results of their study silodosin can be safely used in the treatment of distal ureteric stone in children for decreasing time to stone expulsion, pain episodes, and analgesic requirement. ${ }^{62}$

Fahmy A. Et al. (2017) have conducted a prospective randomized placebo-controlled study including 90 children diagnosed with unilateral, single, radio-opaque distal ureteral stones $<10 \mathrm{~mm}$ in size. ${ }^{63}$ Age ranged between 5.8 and 18 years old. Patients were randomized into three groups; silodosin group $(\mathrm{n}=30)$ received $8 \mathrm{mg}$ silodosin daily, tamsulosin group $(\mathrm{n}=30)$ received $0.4 \mathrm{mg}$ tamsulosin daily and placebo group $(n=30)$ were not given any of the above medications. The stone clearance rates for silodosin, tamsulosin and placebo groups were $78.5,66.6$ and $53.3 \%$, respectively as statisticaly significant. Silodosin was associated with higher stone clearance rates and shorter time to stone clearance as compared to tamsulosin. ${ }^{63}$ Medical expulsive therapy is an ideal treatment for properly selected patients. ${ }^{41}$

\section{Cardiovascular advantages of silodosin}

In an observational study silodosin was added to the LUTS/ BPH treatment of elderly men (age 65 years) using antihypertensive medications between April 2015 and December 2015. The comorbid conditions were categorized by Charlson comorbidity index and the severity of LUTS/BPH has been evaluated by IPSS, MSHQ-Male Sexual Health Questionnaire score. Silodosin has been found safe and effective for management of LUTS in patients who were taking antihypertensive medicines. ${ }^{44}$

Silodosin has minimal cardiovascular effects due to its highly selectivity. ${ }^{45,46}$ Silodosin proved a 583 -fold selectivity for the $\alpha 1 \mathrm{~A}$ versus $\alpha 1 \mathrm{~B}$ subtype and 56 -fold selectivity for the $\alpha 1 \mathrm{~A}$ versus $\alpha 1 \mathrm{D} .^{47}$ Non-selective alpha adrenoceptor antagonists can induce orthostatic hypotension and dizziness. ${ }^{48}$ Silodosin proves superior pharmacologic uroselectivity than an $\alpha$ blocker tamsulosin. Silodosin has 2.5 -fold greater selectivity for the $\alpha 1 \mathrm{~A}$-adrenergic receptor than the $\alpha 1 \mathrm{~B}$ adrenergic receptor and silodosin has 10 -fold greater selectivity for the $\alpha 1 \mathrm{~A}$-adrenergic receptor than the $\alpha 1 \mathrm{D}$-adrenergic receptor. ${ }^{49}$ The minimal selectivity for the $\alpha 1 \mathrm{~B}$ adrenergic receptor, which is mainly engaged in the control of blood pressure, enables silodosin have minimum effects on the cardiovascular system. ${ }^{50,51}$

Cardiovascular diseases are common in potential silodosin user candidates. About 1 in 3 men aged between 65-70 have cardiovascular disease and about $32 \%$ of mortalities were due to cardiovascular disease among people younger than 75 years old. Treatment of BPH with a non-selective adrenergic receptor antagonists may increase dizziness, syncope, hypotension following morbidity and even morbidity. ${ }^{52}$ The other $\alpha 1$ adrenoreceptor blocker with a low patient compliance is prazosin. It requires twice to thrice daily medication and has significant cardiovascular adverse effects, it was also not recommended in the AUA (American Urological Association) guidelines for treatment of $\mathrm{BPH} .{ }^{53}$ Both the Joint National Committee and the AUA recommend separate and proper drug treatment for hypertension and BPH. ${ }^{54}$ ALLHAT trial ( $\mathrm{n}=24,000$ patients) elaborated that both hypertension and BPH should not be treated with doxazosin. ${ }^{55}$

\section{Conclusion}

Silodosin is the most uroselective $\alpha$-blocker. Silodosin has been emphasized in the 2021 European Association of Urology Conservative treatment of non-neurogenic male LUTS guidelines and it has been reported that the hypotensive effect of silodosin is comparable with placebo and has favorable safety and tolerability profile. Dosing of silodosin does not need to be adjusted according to age, concurrent medication with antihypertensives and phosphodiesterase type 5 inhibitors.

\section{Acknowledgments}

None.

\section{Conflicts of interest}

Gokhan Faikoglu, Kubra Saygisever-Faikoglu and Fatmanur Otmar Ozcan are employees of Recordati.

\section{References}

1. Thorner DA, Weiss JP. Benign prostatic hyperplasia: symptoms, symptom scores, and outcome measures. The Urologic Clinics of North America. 2009;36(4):417-429.

2. Berry SJ, Coffey DS, Walsh PC, et al. The development of human benign prostatic hyperplasia with age. The Journal of urology. 1984;132(3):474-479.

3. Shapiro E, Becich MJ, Hartanto V, et al. The relative proportion of stromal and epithelial hyperplasia is related to the development of symptomatic benign prostate hyperplasia. The Journal of urology. 1992;147(5):1293-1297.

4. Rosenberg MT, Witt ES, Miner M, et al. A practical primary care approach to lower urinary tract symptoms caused by benign prostatic hyperplasia (BPH-LUTS). Can J Urol. 2014;21(Suppl 2):12-24.

5. Irwin DE, Milsom I, Hunskaar S, et al. Population-based survey of urinary incontinence, overactive bladder, and other lower urinary tract symptoms in five countries: results of the EPIC study. European Urology. 2006;50(6):1306-1315.

6. Coyne KS, Sexton C, Thompson CL, et al. The prevalence of lower urinary tract symptoms (LUTS) in the USA, the UK and Sweden: results from the Epidemiology of LUTS (EpiLUTS) study. BJU International. 2009;104(3):352-360.

7. Fusco F, Palmieri A, Ficarra V, et al. $\alpha 1$-blockers improve benign prostatic obstruction in men with lower urinary tract symptoms: a systematic review and meta-analysis of urodynamic studies. Eur Urol. 2016;69(6):10911101 .

8. Roehrborn CG, Schwinn DA. Alpha1-adrenergic receptors and their inhibitors in lower urinary tract symptoms and benign prostatic hyperplasia. J Urol. 2004;171(3):1029-1035.

9. Tsuzaka Y, Matsushima H, Kaneko T, et al. Naftopidil vs silodosin in medical expulsive therapy for ureteral stones: a randomized controlled study in Japanese male patients. Int J Urol. 2011;18(11):792-795.

10. Yoshida M, Kudoh J, Homma Y, et al. Safety and efficacy of silodosin for the treatment of benign prostatic hyperplasia. Clin Interv Aging. 2011;6:161-172. 
11. Gül ZG, Kaplan SA. BPH: Why Do Patients Fail Medical Therapy? Curr Urol Rep. 2019;20(7):40.

12. Gravas S, Cornu JN, Gacci MC, et al. Management of Non-neurogenic Male LUTS; 2021.

13. Maxime Rossi, Thierry Roumeguère. Silodosin in the treatment of benign prostatic hyperplasia. Drug Des Devel Ther. 2010;4:291-297.

14. Product Information: RAPAFLO(R) oral capsules, silodosin oral capsules. Allergan USA Inc (per manufacturer): Irvine, CA; 2017.

15. Michel MC, Vrydag W. Alpha1-, alpha2- and beta-adrenoceptors in the urinary bladder, urethra and prostate. Br J Pharmacol. 2006;147 Suppl 2(Suppl 2):S88-S119.

16. Montorsi F. Profile of silodosin. Eur Urol Suppl. 2010;9(4):491-495.

17. Montorsi F. Profile of silodosin. Urologiia. 2013;(2):112-4, 116-7.

18. Marks LS, Gittelman MC, Hill LA, et al. Rapid efficacy of the highly selective $\alpha 1 \mathrm{~A}$-adrenoceptor antagonist silodosin in men with signs and symptoms of benign prostatic hyperplasia: pooled results of 2 phase 3 studies. The journal of urology. 2013;189(6):2634-2640.

19. MacDiarmid SA, Hill LA, Volinn W, et al. Lack of pharmacodynamic interaction of silodosin, a highly selective $\alpha$ la-adrenoceptor antagonist, with the phosphodiesterase- 5 inhibitors sildenafil and tadalafil in healthy men. Urology. 2010;75(3):520-525.

20. Lepor H, Hill LA. Silodosin for the treatment of benign prostatic hyperplasia: pharmacology and cardiovascular tolerability. Pharmacotherapy. 2010;30(12):1303-1312.

21. Capitanio U, Salonia A, Briganti A, et al. Silodosin in the management of lower urinary tract symptoms as a result of benign prostatic hyperplasia: who are the best candidates. International journal of clinical practice. 2013;67(6):544-551.

22. Manohar CMS, Nagabhushana M, Karthikeyan VS, et al. Safety and efficacy of tamsulosin, alfuzosin or silodosin as monotherapy for LUTS in BPH-a double-blind randomized trial. Central European journal of urology. 2017;70(2):148-153.

23. Masciovecchio S, Di Pasquale AB, Ranieri G, et al. Role of silodosin in patients with LUTS/BPE non responding to medical treatment with tamsulosin: a prospective, open-label, pilot study. European review for medical and pharmacological sciences. 2017;21(21): 4941-4945.

24. Chapple CR, Montorsi F, Tammela TL, et al. Silodosin therapy for lower urinary tract symptoms in men with suspected benign prostatic hyperplasia: results of an international, randomized, double-blind, placebo-and active-controlled clinical trial performed in Europe. European urology. 2011;59(3):342-352.

25. Kim TN, Nam JK, Lee KS, et al. Reasons for prescription change of a1-blockers in patients with lower urinary tract symptoms suggestive of benign prostatic hyperplasia. Urology. 2014;84(2):427-432.

26. Yamaguchi K, Aoki Y, Yoshikawa T, et al. Silodosin versus naftopidil for the treatment of benign prostatic hyperplasia: a multicenter randomized trial. International Journal of Urology. 2013;20(12):1234-1238.

27. Yoshihisa Matsukawa, Shun Takai, Yasuhito Funahashi, et al. Long-term efficacy of a combination therapy with an anticholinergic agent and an $\alpha 1$ blocker for patients with benign prostatic enlargement complaining both voiding and overactive bladder symptoms: A randomized, prospective, comparative trial using a urodynamic study. Neurourol Urodyn. 2017;36(3):748-754.

28. Köprü B, Ebiloğlu T, Kaya E, et al. Does preoperative use of silodosin affect the stages of F-URS procedure? Afecta el uso de silodosin preoperatorio los pasos de la ureteroscopia flexible?. Archivos espanoles de urologia. 2020;73(1):47-53.
29. Bhat GS, Shastry A. A prospective double-blind, randomized, placebocontrolled study to evaluate the efficacy of silodosin $8 \mathrm{mg}$ as an ondemand, reversible, nonhormonal oral contraceptive for males: a pilot study. World journal of urology. 2020;38(3):747-751.

30. Cho KJ, Lee JZ, Song YS, et al. Evaluating the Efficacy and Safety of Silodosin on Nocturia in Patients With Benign Prostatic Hyperplasia: A Multicenter, Prospective, Open-label, Single-arm, Phase IV Trial. Urology. 2018;121:153-157.

31. Moon KH, Park CH, Jung HC, et al. A 12-Week, Open Label, MultiCenter Study to Evaluate the Clinical Efficacy and Safety of Silodosin on Voiding Dysfunction in Patients with Neurogenic Bladder. LUTS: Lower Urinary Tract Symptoms. 2015;7(1):27-31.

32. Imperatore V, Fusco F, Creta M, et al. Medical expulsive therapy for distal ureteric stones: tamsulosin versus silodosin. Archivio Italiano di Urologia e Andrologia. 2014;86(2):103-107.

33. Sato Y, Otani T, Amano T, et al. Silodosin versus naftopidil in the treatment of premature ejaculation: A prospective multicenter trial. International journal of urology: official journal of the Japanese Urological Association. 2017;24(8):626-631.

34. Roehrborn CG, Lepor H, Kaplan SA. Retrograde ejaculation induced by silodosin is the result of relaxation of smooth musculature in the male urogenital tracts and is associated with greater urodynamic and symptomatic improvements in men LUTS secondary to BPH. The Journal of Urology. 2009;181(4S):694-695.

35. Sertkaya Z, Ozkaya F. Silodosin has nocebo effect on sexual adverse effects: a randomized controlled trial. The Eurasian journal of medicine. 2019;51(3):277-279.

36. Fusco F, Creta M, Longo N, et al. Silodosin $8 \mathrm{mg}$ improves benign prostatic obstruction in Caucasian patients with lower urinary tract symptoms suggestive of benign prostatic enlargement: results from an explorative clinical study. BMC urology. 2018;18(1):12.

37. Yilmaz E, Batislam E, Basar MM, et al. The comparison and efficacy of 3 different $\alpha 1$-adrenergic blockers for distal ureteral stones. The journal of urology. 2005;173(6):2010-2012.

38. Dell'Atti L. Silodosin versus tamsulosin as medical expulsive therapy for distal ureteral stones: a prospective randomized study. Urologia. 2015;82(1):54-57.

39. Kumar S, Jayant K, Agrawal MM, et al. Role of tamsulosin, tadalafil, and silodosin as the medical expulsive therapy in lower ureteric stone: a randomized trial (a pilot study). Urology. 2015;85(1):59-63.

40. Wang CJ, Tsai PC, Chang CH. Efficacy of Silodosin in Expulsive Therapy for Distal Ureteral Stones: A Randomized Double-blinded Controlled Trial. Urology journal. 2016;13(3):2666-2671.

41. Kyle DW, Ilya G, Jorge Gutierrez. Medical expulsive therapy. Indian J Urol. 2014;30(1):60-64.

42. Gharib T, Mohey A, Fathi A, et al. Comparative Study between Silodosin and Tamsulosin in Expectant Therapy of Distal Ureteral Stones. Urologia internationalis. 2018;101(2):161-166.

43. Bayar G, Yavuz A, Cakmak S, et al. Efficacy of silodosin or mirabegron in medical expulsive therapy for ureteral stones: a prospective, randomizedcontrolled study. International urology and nephrology. 2020;52(5):835840

44. Choi WS, Cho MC, Lee JW, et al. Efficacy and safety of silodosin in the treatment of lower urinary tract symptoms in elderly men taking antihypertensive medications. Prostate international. 2017;5(3):113-118.

45. Murata S, Taniguchi T, Takahashi M, et al. Tissue selectivity of KMD3213 , an $\alpha 1$-adrenoceptor antagonist, in human prostate and vasculature. The Journal of urology. 2000;164(2):578-583. 
46. Yamada S, Kato Y, Okura T, et al. Prediction of $\alpha 1$-adrenoceptor occupancy in the human prostate from plasma concentrations of silodosin, tamsulosin and terazosin to treat urinary obstruction in benign prostatic hyperplasia. Biological and Pharmaceutical Bulletin. 2007;30(7):1237-1241.

47. Shibata K, Foglar R, Horie K, et al. KMD-3213, a novel, potent, alpha 1a-adrenoceptor-selective antagonist: characterization using recombinan human alpha 1-adrenoceptors and native tissues. Molecular Pharmacology. $1995 ; 48(2): 250-258$.

48. Humphrey SJ, McCall RB. A rat model for predicting orthostatic hypotension during acute and chronic antihypertensive drug therapy. Journal of pharmacological methods. 1982;7(1):25-34.

49. Lepor H. Alpha-blockers for the treatment of benign prostatic hyperplasia Urologic Clinics. 2016;43(3):311-323.

50. Martin DJ, Angel I, Arbilla S. Functional uroselectivity. European urology. 1998;33(Suppl 2):12-18.

51. Roehrborn CG. Efficacy of $\alpha$-adrenergic receptor blockers in the treatment of male lower urinary tract symptoms. Reviews in urology. 2009;11(Suppl 1):S1-8.

52. Fine SR, Ginsberg P. Alpha-adrenergic receptor antagonists in older patients with benign prostatic hyperplasia: issues and potential complications. Journal of the American Osteopathic Association. 2008;108(7):333-337.

53. DiPiro J, Yee G, Posey L, et al. Pharmacotherapy a Pathophysiologic Approach. 11th ed. McGraw-Hill; 2020.

54. American Geriatrics Society 2012 Beers Criteria Update Expert Panel. American Geriatrics Society updated Beers Criteria for potentially inappropriate medication use in older adults. $J$ Am Geriatr Soc. 2012;60(4):616-631.
55. Whelton PK, Carey RM, Aronow WS. Detection, evaluation, and management of high blood pressure in adults: a Report of the American College of Cardiology/American heart Association Task force on clinical practice guidelines. Hypertension. 2018;71(6):1269-1324.

56. Collaborative Research Group. Major cardiovascular events in hypertensive patients randomized to doxazosin vs chlorthalidone: the antihypertensive and lipid-lowering treatment to prevent heart attack trial (ALLHAT). JAMA. 2000;283(15):1967-1975.

57. Boeri L, Capogrosso P, Ventimiglia E, et al. Clinically meaningful improvements in LUTS/BPH severity in men treated with silodosin plus hexanic extract of Serenoa repens or silodosin alone. Scientific reports. 2017;7(1):15179.

58. Jae Hung Jung, Jiye Kim, Roderick MacDonald, et al. Silodosin for the treatment of lower urinary tract symptoms in men with benign prostatic hyperplasia. Cochrane Database Syst Rev. 2017;11(11):CD012615.

59. Roderick MacDonald, Michelle Brasure, Philipp Dahm, et al. Efficacy of newer medications for lower urinary tract symptoms attributed to benign prostatic hyperplasia: a systematic review. Aging Male. 2019;22(1):1-11.

60. Soliman MG, El-Gamal O, El-Gamal S, et al. Silodosin versus Tamsulosin as Medical Expulsive Therapy for Children with Lower-Third Ureteric Stones: Prospective Randomized Placebo-Controlled Study. Urol Int. 2021;105(7-8):568-573.

61. Elgalaly Hazem, Eliwa Ahmed, Seleem Mohamed, et al. Silodosin in the treatment of distal ureteric stones in children: A prospective, randomised, placebo-controlled study. Arab J Urol. 2017;15(3):194-198.

62. Fahmy Ahmed, Hazem Rhasad, Amr Kamal, et al. MP90-18 silodosin for medical expulsive therapy in children with distal ureteral stone: a prospective randomized, placebo-controlled, single blind study study. The Journal of Urology. 2017;197(4S):e1217. 